\title{
Lidil
}

Revue de linguistique et de didactique des langues

\section{Fayol M., Jaffré J.-P., Orthographier, PUF, 2008, collection « Apprendre».}

\section{Claudie Péret}

\section{(2) OpenEdition}

1 Journals

Édition électronique

URL : http://journals.openedition.org/lidil/2771

DOI : 10.4000/lidil.2771

ISSN : 1960-6052

Éditeur

UGA Éditions/Université Grenoble Alpes

\section{Édition imprimée}

Date de publication : 1 décembre 2008

Pagination : 139

ISBN : 978-2-84310-130-4

ISSN : $1146-6480$

\section{Référence électronique}

Claudie Péret, « Fayol M., Jaffré J.-P., Orthographier, PUF, 2008, collection « Apprendre ». », Lidil [En ligne], 38 | 2008, mis en ligne le 01 juin 2010, consulté le 21 septembre 2020. URL : http:// journals.openedition.org/lidil/2771; DOI : https://doi.org/10.4000/lidil.2771

Ce document a été généré automatiquement le 21 septembre 2020.

(C) Lidil 


\title{
Fayol M., Jaffré J.-P., Orthographier, PUF, 2008, collection « Apprendre ».
}

\author{
Claudie Péret
}

L'ouvrage co-signé du linguiste Jean-Pierre Jaffré et du psycholinguiste Michel Fayol, réunit les contributions de ces deux chercheurs à qui l'on doit des avancées dans la compréhension du système orthographique du français pour le premier et dans la façon dont l'individu se l'approprie pour le second. D'où le titre mettant l'accent sur l'acte de produire la langue française en respectant son orthographe, Orthographier.

1 Les six chapitres rédigés par Jean-Pierre Jaffré permettent de différencier orthographe et écriture et situent l'orthographe française en diachronie en montrant l'impact des facteurs non linguistiques (politiques et religieux par exemple) sur son évolution et en mettant en évidence les parts respectives de la phonographie et de la sémiographie dans le système actuel. L'ouvrage faisant la synthèse des travaux de l'auteur dans le domaine, présente ce sur quoi ses derniers travaux ont porté : l'importance de la dimension sémiographique dans l'efficacité de la compréhension de l'écrit. Le descriptif des états antérieurs de l'orthographe française comme celui de systèmes orthographiques d'autres langues met en évidence la complexité du système français, relativise l'intangibilité de la norme et rend évidente la difficulté que représente son apprentissage.

2 C'est cet aspect que développe Michel Fayol dans les six chapitres de la deuxième partie. L'apport de la psycholinguistique dans le domaine permet de prendre conscience des difficultés rencontrées par le scripteur en langue française pour gérer tous les aspects de l'orthographe et des différents mécanismes cognitifs qu'utilise l'expert pour résoudre les problèmes qu'il rencontre en lecture comme en écriture. La contribution de l'auteur est là aussi en lien avec ses derniers travaux sur la place de la connaissance et de l'application des règles d'une part et des récupérations d'instances d'autre part. Comment se construit la compétence orthographique? Que faut-il privilégier au moment de l'apprentissage? Quelles conséquences pour l'enseignement? Même si l'ouvrage n'apporte pas de réponse définitive à ces questions (le pourrait-il ?), 
il permet d'apporter des éléments pouvant éclairer le praticien de l'enseignement de l'orthographe.

3 Sous une apparente juxtaposition, les deux apports, linguistique et psycholinguistique, se répondent grâce aux renvois effectués par les auteurs aux différents chapitres de l'ouvrage. Les chercheurs respectent en cela le cahier des charges que la collection "Apprendre » s'est fixé : établir des ponts entre les résultats de la recherche et les questions de pratiques d'enseignement. La collection a aussi un souci de lisibilité pour s'adresser à un public de non spécialistes mais professionnels impliqués dans l'enseignement de l'orthographe. C'est ainsi que des encadrés "J'ai lu», "Histoires d'orthographes ", "Drôle d'expérience ", " Nouveaux usages » ou " $\mathrm{Vu}$ ailleurs » viennent illustrer de façon concrète les propos des auteurs. D’autres encadrés intitulés «Des pistes pour l'école» sont plus directement orientés vers les professionnels. Le volume se termine par un glossaire qui aide le non spécialiste à se repérer dans la terminologie du domaine. Cependant le souci de présenter les apports récents de la recherche pour les professionnels est au détriment des références précises concernant les derniers travaux des auteurs ou des travaux auxquels ils se réfèrent, frustrant celui qui voudrait voir dans cette synthèse un guide pour pousser plus loin ses lectures. Le praticien de l'enseignement de l'orthographe peut quant à lui trouver insuffisantes en nombre et en contenu les "pistes pour l'école». Le renvoi à des ouvrages de didacticiens serait alors nécessaire. Cette réserve n'est pas à mettre sur le compte des auteurs qui ont suivi les lignes imposées par l'éditeur.

\section{AUTEUR \\ CLAUDIE PÉRET}

LIDILEM, IUFM de Grenoble 